\title{
TESTING BRCA OR MULTIGENIC PANELS AFTER MEDICAL RECOMMENDATION
}

\author{
Sabas Carlos Vieira', Danilo Rafael da Silva Fontinele², Ana Lúcia Nascimento Araújo \\ 'Oncocenter; Oncobem - Teresina (PI) - Brazil. \\ universidade Estadual do Piauí - Teresina (PI), Brazil.
}

Introduction: Hereditary breast cancer represents about $10 \%$ of breast cancer cases. Risk factors for hereditary breast cancer are family history of breast cancer, ovarian cancer, breast cancer in men, among others, especially at an early age and bilateral tumors. Several hereditary syndromes have been described in relation to breast cancer, the most frequent being the Breast, Ovarian and Pancreatic Cancer Syndrome, determined by mutations in the BRCA 1 and 2 genes. In Brazil, the difficulty of accessing the genetic test of the public and private systems is still a reality, mainly due to elevate costs. Objectives: To raise patients' adherence to perform the BRCA test or multigene panels after medical recommendation at a private clinic in Piauí. Method: This is a retrospective study, evaluating medical records of patients seen in the period from 1999 to 2019. This study included all patients who had a recommendation to perform the BRCA mutation research or multigene panels. The study was approved by the ethics and research committee of Universidade Federal do Piauí, Opinion n. 2.817.502. Results: 164 patients who had a recommendation for genetic testing were studied. There were 162 female patients $(98.7 \%)$ and mean age was 48.3 years. As for the presence of cancer, breast cancer was the most frequent one with 82 (79.6\%) cases; as for survival, 5 (3.0\%) of the cases evolved to death. 86 (52.4\%) of the patients underwent the test, with mean gap between the recommendation and the test itself of 2.3 years. Of those who performed the tests, 23 (26.7\%) had mutations in the BRCA gene, 15 (65.2\%) in BRCA1, and 8 (34.8\%) in BRCA2. Conclusions: $52.4 \%$ of the patients recommended for genetic testing performed the research. Of those who performed the tests, $26.7 \%$ had mutations in the BRCA gene, $65.2 \%$ in BRCA1, and 34.8\% in BRCA2. 